\title{
Theory of transmission through disordered superlattices
}

\author{
Wacker, Andreas; Hu, Ben Yu-Kuang
}

Published in:

Physical Review B

Link to article, DOI:

10.1103/PhysRevB.60.16039

Publication date:

1999

Document Version

Publisher's PDF, also known as Version of record

Link back to DTU Orbit

Citation (APA):

Wacker, A., \& Hu, B. Y-K. (1999). Theory of transmission through disordered superlattices. Physical Review B, 60(23), 16039-16049. https://doi.org/10.1103/PhysRevB.60.16039

\section{General rights}

Copyright and moral rights for the publications made accessible in the public portal are retained by the authors and/or other copyright owners and it is a condition of accessing publications that users recognise and abide by the legal requirements associated with these rights.

- Users may download and print one copy of any publication from the public portal for the purpose of private study or research.

- You may not further distribute the material or use it for any profit-making activity or commercial gain

- You may freely distribute the URL identifying the publication in the public portal

If you believe that this document breaches copyright please contact us providing details, and we will remove access to the work immediately and investigate your claim. 


\title{
Theory of transmission through disordered superlattices
}

\author{
Andreas Wacker \\ Institut für Theoretische Physik, Technische Universität Berlin, Hardenbergstrasse 36, 10623 Berlin, Germany \\ Ben Yu-Kuang Hu \\ Mikroelektronik Centret, Danmarks Tekniske Universitet, DK-2800 Lyngby, Denmark \\ and Department of Physics, 250 Buchtel Commons, University of Akron, Akron, Ohio 44325-4001*
}

(Received 1 April 1999)

\begin{abstract}
We derive a theory for transmission through disordered finite superlattices in which the interface roughness scattering is treated by disorder averaging. This procedure permits efficient calculation of the transmission through samples with large cross sections. These calculations can be performed utilizing either the Keldysh or the Landauer-Büttiker transmission formalisms, both of which yield identical equations. For energies close to the lowest miniband, we demonstrate the accuracy of the computationally efficient Wannier-function approximation. Our calculations indicate that the transmission is strongly affected by interface roughness and that information about scale and size of the imperfections can be obtained from transmission data.
\end{abstract}

[S0163-1829(99)01946-3]

\section{INTRODUCTION}

Semiconductor superlattices continue to attract substantial interest both among fundamental and applied researchers. One motivating factor is the possibility of tailoring the miniband structure ${ }^{1-3}$ for device purposes. Furthermore, a large variety of other physical phenomena such as the formation of Wannier-Stark ladders, ${ }^{4}$ negative differential conductance, ${ }^{5}$ and Bloch oscillations ${ }^{6}$ can be observed in superlattices.

The presence of minibands has been probed directly by investigating the transmission of ballistic electrons through short semiconductor superlattices. ${ }^{7,8}$ In recent experiments the quenching of the miniband structure by an applied electric field was also demonstrated. ${ }^{9}$ Comparison of further experiments with theoretical calculations indicated a strong influence of scattering on the transmission, and it was argued that interface roughness might cause significant deviations from pure ballistic transmission through the sample. ${ }^{10,11}$

A good understanding of the transmission characteristics through short superlattices is important as these structures are used as energy filters. For example, in quantum cascade lasers, superlattice filters are used to selectively populate the upper energy level of the active region. ${ }^{12}$ The most straightforward way to calculate the transmission through a superlattice is the transfer-matrix method. ${ }^{13}$ Alternatively, the Schrödinger equation of the superlattice can be solved directly. These methods typically assume homogeneity in the direction perpendicular [the $(x, y)$ plane] to the superlattice. The momenta in the $(x, y)$ plane are then good quantum numbers and decouple from the superlattice direction, reducing the problem to a one-dimensional calculation; see, e.g., Ref. 14. The one-dimensional calculation can handle fluctuations ${ }^{15}$ in the well or barrier thickness. However, real samples also exhibit a lack of periodicity in the $(x, y)$ plane due to the presence of impurities and interface roughness. This can change the transport properties essentially, as the states with different parallel momenta couple to each other. This $(x, y)$ plane inhomogeneity can be tackled by solving the Schrödinger equation on a mesh for the full three dimensional structure. ${ }^{16}$ Alternatively, the method of Green functions, based on Ref. 17, may be used (see Ref. 18 for an easily accessible presentation of the method). Recently such an approach has been presented for a full calculation of the current through a resonant tunneling diode where both interface roughness and phonon scattering have been taken into account. ${ }^{19}$ However, these simulations use a fine grid and are hence unsuitable for longer structures such as superlattices consisting of many wells, since the number of grid points increases dramatically.

In this paper we propose a method for such calculations which significantly reduces the computational complexity. We treat the inhomogeneity in the $(x, y)$ plane by averaging over disorder configurations. The number of grid points in the $z$ direction is reduced significantly by restricting to the basis set to the Wannier functions localized in the wells. This Wannier approximation is shown to be valid near the resonance condition if the energy gap between the minibands is large compared to the bias and the widths of the minibands themselves. We compare our results to calculations on a finite grid and find good agreement. Our method has the advantage that it corresponds to infinitely large cross sections and hence, unlike the finite-grid calculations, does not show configuration dependent fluctuations.

The paper is organized as follows. We present the general model within which our calculations are performed in Sec. II. In Sec. III, we describe the approximations which allow us to perform practical calculations of extended superlattice structures. Our results are presented in Sec. IV and we conclude with a summary. Appendix A shows the equivalence of the Landauer-Büttiker transmission formalism with the approach by nonequilibrium Green functions for the case of impurity averaging. In Appendix B we justify the approximations used in Sec. III. As many different symbols appear in this paper, for easy reference we display the frequently used ones in Table I. 
TABLE I. Table of various symbols used in this paper.

\begin{tabular}{|c|c|}
\hline$l, l^{\prime}$ & lead index \\
\hline$\alpha, \beta$ & sub-band index (in leads) \\
\hline$C$ & central region \\
\hline$\left|\phi_{l \alpha q}\right\rangle$ & state in lead $l$, subband $\alpha$ with wave number $q$ \\
\hline$\left|\phi_{C, i}\right\rangle$ & state in central region \\
\hline$i, j$ & index of central region states \\
\hline$n, m$ & index of quantum well \\
\hline$f_{l \alpha}(E)$ & distribution function of lead $l$, sub-band $\alpha$ \\
\hline G & $\begin{array}{l}\text { Green function in central region (matrix with respect } \\
\text { to } i, j \text { or } n, m \text { ) }\end{array}$ \\
\hline$\Sigma$ & self-energy in central region \\
\hline$\Gamma$ & $i\left(\Sigma^{\mathrm{ret}}-\boldsymbol{\Sigma}^{\mathrm{adv}}\right)$ \\
\hline ret, adv, $<$ & $\begin{array}{l}\text { retarded, advanced, " }<\text {,' component of Keldysh } \\
\text { functions }\end{array}$ \\
\hline$\overline{\mathbf{G}}$ & impurity average (in central region) of $\mathbf{G}$ \\
\hline k & $\begin{array}{l}\text { wave vector in }(x, y) \text { plane (i.e., plane } \| \text { to } \\
\text { superlattice interfaces) }\end{array}$ \\
\hline$E_{q}$ & $\begin{array}{l}\text { "longitudinal" kinetic energy in lead } \hbar^{2} q^{2} / 2 m \\
\text { (assumed } l, \alpha \text { independent) }\end{array}$ \\
\hline$E_{l \alpha}$ & "transverse" kinetic energy of lead $l$, subband $\alpha$ \\
\hline$E^{a}$ & energy of middle of miniband in central region \\
\hline $\mathrm{v}_{q}$ & longitudinal velocity of particle $\hbar q / m$ in lead \\
\hline$U_{l}$ & applied potential in lead $l$ \\
\hline$L_{l}$ & length of lead $l$ \\
\hline$J_{l \alpha}$ & current into central region from $l, \alpha$ \\
\hline$e$ & charge of the electron $(e<0)$ \\
\hline
\end{tabular}

\section{GENERAL FORMALISM}

In this paper, we study transport through a superlattice contacted to external voltage sources via leads. We model the superlattice as an active central region coupled to noninteracting lead regions. This is the general approach described in Refs. 17, 18, 20, 21, and 22. In this section we briefly review this approach, introduce our notation, and discuss the issue of impurity averaging.

We divide the sample into a central region $\mathrm{C}$ and lead regions, indexed by $l$. The Hamiltonian is

$$
\hat{H}=\hat{H}_{C}+\sum_{l} \hat{H}_{l}+\hat{H}_{l C}+\hat{H}_{l C}^{\dagger} .
$$

Here $\hat{H}_{C}$ and $\hat{H}_{l}$ are the terms for the central structure and leads, respectively, and $\hat{H}_{l C}$ is the coupling term from the center to the lead $l$. In this paper, we ignore electron-electron interactions beyond Hartree so all the above terms are singleparticle-like.

The central structure has states with the wave function $\phi_{C, j}(\vec{r})$, where $j$ is the eigenstate index. We assume that each lead $l$ is disorder-free so that the eigenstates can be separated into transverse and longitudinal parts, $\phi_{l \alpha q}(\vec{r})$ $=\chi_{l \alpha}(\mathbf{r}) \varphi_{q}^{l}(z)$, where $z$ is the spatial coordinate in the direction towards the central structure and $\mathbf{r}$ is a two-dimensional vector perpendicular to $z$. The index $\alpha$ numbers the modes within a given lead. The index $q$ denotes the behavior far away from the central region where $\varphi_{q}^{l}(z) \sim e^{i q z}$ is assumed.

\section{A. Green functions and current through structure}

The current through a structure can be determined by the Green function of the structure in the presence of coupling to the leads, given by a matrix $\mathbf{G}$ with matrix elements

$$
\begin{gathered}
G_{i j}^{<}\left(t, t^{\prime}\right)=i\left\langle\hat{c}_{j}^{\dagger}\left(t^{\prime}\right) \hat{c}_{i}(t)\right\rangle, \\
G_{i j}^{\operatorname{ret}} \operatorname{adv}\left(t, t^{\prime}\right)=\mp i\left\langle\left\{\hat{c}_{i}(t), \hat{c}_{j}^{\dagger}\left(t^{\prime}\right)\right\}\right\rangle \theta\left[ \pm\left(t-t^{\prime}\right)\right] .
\end{gathered}
$$

Here $\hat{c}_{i}^{\dagger}\left(\hat{c}_{i}\right)$ are fermion creation (annihilation) operators of states $\phi_{C, i}$ in the central region, and $\{\ldots, \ldots\}$ denote anticommutators.

In the following we consider time-independent problems, so that $\mathbf{G}$ only depends on $t-t^{\prime}$, and we work in energyspace by Fourier transforming $\mathbf{G}$ with respect to $t-t^{\prime}$. The net current from mode $\alpha$ in lead $l$ into the structure is given by $^{20,22}$

$$
\begin{aligned}
J_{l \alpha}= & 2 \frac{i e}{\hbar} \int \frac{d E}{2 \pi} \operatorname{Tr}\left(\boldsymbol { \Gamma } _ { l \alpha } ( E ) \left\{\mathbf{G}^{<}(E)+f_{l \alpha}(E)\right.\right. \\
& \left.\left.\times\left[\mathbf{G}^{\mathrm{ret}}(E)-\mathbf{G}^{\mathrm{adv}}(E)\right]\right\}\right) .
\end{aligned}
$$

Here $f_{l \alpha}(E)$ gives the occupation of a state with energy $E$ in lead $l$ for the mode $\alpha, e<0$ is the charge of the electron, and $\boldsymbol{\Gamma}_{l, \alpha}$ is a parameter describing the coupling between the states in the central region and the leads [see Eq. (6) below]. The factor of 2 is for spin.

To describe transmission through the superlattice, we need to obtain expressions for the right-hand side of Eq. (3). We do so as follows. We first define $\hat{H}_{C, 0}$ and $\hat{H}_{C}^{\prime}$, as the ordered, solvable part and the disordered part of the central region Hamiltonian, respectively, and $\hat{H}_{C}=\hat{H}_{C, 0}+\hat{H}_{C}^{\prime}$. The retarded Green function for the structure is determined by the equation (see Ref. 22, Chap. 12)

$$
\left(E-\mathbf{H}_{C, 0}-\boldsymbol{\Sigma}_{C}^{\mathrm{ret}}-\sum_{l \alpha} \boldsymbol{\Sigma}_{l \alpha}^{\mathrm{ret}}(E)\right) \mathbf{G}^{\mathrm{ret}}(E)=\mathbf{1} .
$$

The term $\Sigma_{C}^{\text {ret }}$ is the irreducible self-energy due to $\hat{H}_{C}^{\prime}$. In cases where $\hat{H}_{C}^{\prime}$ contains interparticle interactions, $\Sigma_{C}^{\text {ret }}$ is often very difficult to calculate; however, for static disorder, simply $\boldsymbol{\Sigma}_{C}^{\text {ret }}=\mathbf{H}_{C}^{\prime}$. The term $\boldsymbol{\Sigma}_{l \alpha}^{\text {ret }}(E)$ gives the self-energy contributions due to the coupling of the central region to lead $l$ and mode $\alpha$,

$$
\begin{aligned}
\Sigma_{l \alpha, i j}^{\mathrm{ret}}(E)= & \sum_{q}\left\langle\phi_{C, i}\left|\hat{H}_{l C}^{\dagger}\right| \phi_{l \alpha q}\right\rangle\left\langle\phi_{l \alpha q}\left|\hat{H}_{l C}\right| \phi_{C, j}\right\rangle g_{l \alpha q}^{\mathrm{ret}}(E) \\
= & \frac{L_{l}}{2 \pi} \int_{0}^{\infty} d E_{q} \frac{2}{\hbar \mathrm{v}_{q}}\left\langle\phi_{C, i}\left|\hat{H}_{l C}^{\dagger}\right| \phi_{l \alpha q}\right\rangle \\
& \times\left\langle\phi_{l \alpha q}\left|\hat{H}_{l C}\right| \phi_{C, j}\right\rangle g_{l \alpha q}^{\mathrm{ret}}(E),
\end{aligned}
$$


where we have taken the continuum limit $\Sigma_{q}$ $\rightarrow L_{l} / 2 \pi \int_{-\infty}^{\infty} d q\left(L_{l}\right.$ is the length of lead $\left.l\right)$. The factor 2 results from the two possible values $\pm q$ for a given energy $E_{q}, \quad g_{l \alpha q}^{\text {ret }}(E)=1 /\left(E-E_{q}-E_{l \alpha}+i 0^{+}\right)$is the free-particle Green function of the lead in absence of the central region,
$E_{q}=\hbar^{2} q^{2} / 2 m^{*}, \mathrm{v}_{q}=\hbar q / m^{*}$ and $E_{l, \alpha}$ is the lateral energy of the mode $\alpha$. Here $m^{*}$ is the effective electron mass. Note that $G_{i j}^{\mathrm{adv}}(E)=\left[G_{j i}^{\mathrm{ret}}(E)\right]^{*}$, since we have a time-independent system.

The coupling parameter $\boldsymbol{\Gamma}_{l \alpha}$ is defined by

$$
\begin{aligned}
\Gamma_{l \alpha, i j}(E) & =i\left[\sum_{l \alpha, i j}^{\mathrm{ret}}(E)-\Sigma_{l \alpha, i j}^{\mathrm{adv}}(E)\right] \\
& \equiv \frac{2 L_{l}\left\langle\phi_{C, i}\left|\hat{H}_{l C}^{\dagger}\right| \phi_{l \alpha q\left(E-E_{l \alpha}\right)}\right\rangle\left\langle\phi_{l \alpha q\left(E-E_{l \alpha}\right)}\left|\hat{H}_{l C}\right| \phi_{C, j}\right\rangle}{\hbar \mathrm{v}_{q\left(E-E_{l \alpha}\right)}} \Theta\left(E-E_{l \alpha}\right),
\end{aligned}
$$

where $q(\mathcal{E})=\sqrt{2 m^{*} \mathcal{E}} / \hbar . \Theta(x)$ is the Heaviside function with $\Theta(x)=1$ for $x \geqslant 0$ and $\Theta(x)=0$ for $x<0$. Note that $\Gamma_{l \alpha, i j}(E)=0$ for $E<E_{l \alpha}$ since there are no propagating states into which the central-region states can tunnel.

$\mathbf{G}^{<}(E)$ can be obtained by the Keldysh relation ${ }^{23}$

$$
\mathbf{G}^{<}(E)=\mathbf{G}^{\mathrm{ret}}(E) \mathbf{\Sigma}^{<}(E) \mathbf{G}^{\mathrm{adv}}(E),
$$

where

$$
\boldsymbol{\Sigma}^{<}(E)=\boldsymbol{\Sigma}_{C}^{<}(E)+\sum_{l \alpha} \boldsymbol{\Sigma}_{l \alpha}^{<}(E)
$$

Here, $\boldsymbol{\Sigma}_{C}^{<}$is the self-energy resulting from scattering inside the structure. For a fixed disorder potential, this term is identically zero. The term $\boldsymbol{\Sigma}_{l \alpha}^{<}(E)$ is the self-energy due to the presence of the coupling to the leads,

$$
\begin{aligned}
\Sigma_{l \alpha, i j}^{<}(E) & =\sum_{q}\left\langle\phi_{C, i}\left|\hat{H}_{l C}^{\dagger}\right| \phi_{l \alpha q}\right\rangle\left\langle\phi_{l \alpha q}\left|\hat{H}_{l C}\right| \phi_{C, j}\right\rangle g_{l \alpha q}^{<}(E) \\
& =i \Gamma_{l \alpha, i j}(E) f_{l \alpha}(E),
\end{aligned}
$$

where we have used $g_{l \alpha q}^{<}(E)=-2 i f_{l \alpha}(E) \operatorname{Im}\left\{g_{l \alpha q}^{\text {ret }}(E)\right\}$, The occupation function $f_{l \alpha}(E)$ in lead $l$ and is given by the externally imposed conditions. Usually, the leads are assumed to be in thermal equilibrium and hence a Fermi distribution with chemical potential $\mu_{l}$, independent of $\alpha$, is used. In contrast, the different modes can be populated individually by injection, as discussed later, so that we want to keep the full function $f_{l \alpha}(E)$.

\section{B. Relation to the Landauer-Büttiker approach}

The Landauer-Büttiker approach has been used extensively to study transmission through mesoscopic structures, and consequently many people are familiar with the formalism. As the Keldysh formulation is not as widely known, in this subsection we demonstrate the equivalence of the two approaches for transport through a system with static disorder.

The retarded and advanced Green functions can be expressed in terms of $\Gamma$ via

$$
\mathbf{G}^{\mathrm{ret}}(E)-\mathbf{G}^{\mathrm{adv}}(E)=-i \mathbf{G}^{\mathrm{ret}}(E) \boldsymbol{\Gamma}(E) \mathbf{G}^{\mathrm{adv}}(E),
$$

where the total scattering rate $\boldsymbol{\Gamma}$ has two contributions:

$$
\boldsymbol{\Gamma}(E)=i\left[\boldsymbol{\Sigma}_{C}^{\mathrm{ret}}(E)-\boldsymbol{\Sigma}_{C}^{\mathrm{adv}}(E)\right]+\sum_{l \alpha} \boldsymbol{\Gamma}_{l \alpha}(E)
$$

resulting from scattering inside the structure and transitions into the leads. If the scattering within the structure itself is purely elastic and is treated in a particular fixed configuration as a potential in Eq. (4), then $\boldsymbol{\Sigma}_{C}^{\mathrm{ret}}=\mathbf{H}_{C}^{\prime}$ and $\boldsymbol{\Sigma}_{C}^{<}(E)=0$; hence we may insert Eqs. (7) and (10) into Eq. (3) and find the Landauer-Büttiker expression ${ }^{20,24}$

$$
J_{l \alpha}=2 \frac{e}{\hbar} \int \frac{d E}{2 \pi} \sum_{l^{\prime} \beta} T_{l \alpha \leftarrow l^{\prime} \beta}(E)\left[f_{l \alpha}(E)-f_{l^{\prime} \beta}(E)\right]
$$

(factor of 2 for spin) with the transmission matrix

$$
T_{l \alpha \leftarrow l^{\prime} \beta}(E)=\operatorname{Tr}\left\{\boldsymbol{\Gamma}_{l \alpha}(E) \mathbf{G}^{\mathrm{ret}}(E) \boldsymbol{\Gamma}_{l^{\prime} \beta}(E) \mathbf{G}^{\mathrm{adv}}(E)\right\} .
$$

(There are several alternate ways to derive this result; e.g., Ref. 18 uses spatial discretization.) Note that Eq. (13) does not hold if the scattering process is inelastic or the elastic scattering by static disorder is described by a self-energy obtained by configuration averaging. In both cases $\boldsymbol{\Sigma}_{C}^{<}(E)$ $\neq 0$ in contrast to the assumption leading to Eq. (12).

\section{Impurity averaging}

Equation (13) is exact for a given configuration of impurities and roughness, i.e., for a specific $\mathbf{H}_{C}^{\prime}$. However, obtaining the transmission by simulating individual configurations is not computationally efficient, and hence it is advantageous to average over impurity configurations. In particular, such a procedure reestablishes symmetries which are broken by specific impurity configurations, thus simplifying the calculation significantly.

After impurity averaging, we obtain

$$
\overline{\mathbf{G}}^{\mathrm{ret}}(E)=\left[E+i 0^{+}-\mathbf{H}_{C, 0}-\overline{\boldsymbol{\Sigma}}_{C}^{\mathrm{ret}}(E)-\sum_{l} \boldsymbol{\Sigma}_{l}^{\mathrm{ret}}(E)\right]^{-1},
$$

where the overlines indicate averages over disorder configurations in the central region. Note that the disorder averaging introduces nonzero self-energies $\overline{\boldsymbol{\Sigma}}_{C}^{\text {ret }}(E)$ and $\overline{\boldsymbol{\Sigma}}_{C}^{<}(E)$. As $\overline{\boldsymbol{\Sigma}}_{C}^{<}(E) \neq 0$ one cannot simply use Eq. (13) with the $G$ 's re- 


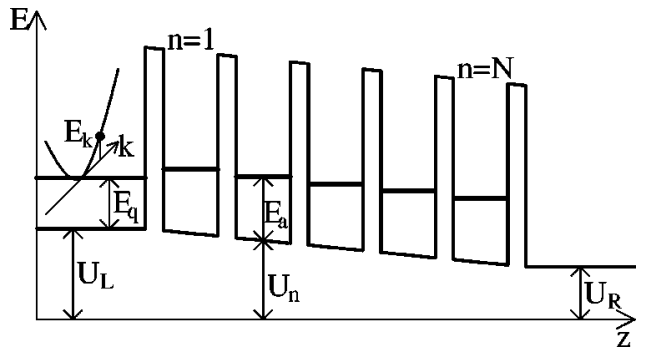

FIG. 1. Sketch of the structure considered. The energy levels are indicated for $E_{k}=0$, which has to be added for finite parallel momentum $k$.

placed by $\bar{G}$. In order to describe configuration-averaged elastic scattering within the transmission formalism, the averaging must be performed for the total transmission matrix in Eq. (13), and not just on the individual $G^{\text {ret }}$ and $G^{\text {adv }}$. This procedure is similar to the calculation of bulk conductivities using the Kubo formula, where it is crucial to include vertex corrections which fulfill the Ward identity (see, e.g., Ref. 25). We perform such a calculation in Appendix A for the superlattice structure discussed in Sec. III. We use the selfconsistent Born approximation for the scattering and therefore the appropriate vertex function is the so-called ladder approximation.

The application of the more general Keldysh approach to calculate the current in the configuration averaged case is more straightforward, in that one can replace the $G$ by $\bar{G}$ in Eq. (3). Therefore, in order to evaluate the current, we need $\overline{\mathbf{G}}^{<}(E)$ and $\overline{\mathbf{G}}^{\text {ret }}(E)$. The general iterative procedure for computing these is as follows. First the self-energies $\boldsymbol{\Sigma}_{l}^{\text {ret }}(E)$ and $\boldsymbol{\Sigma}_{l}^{<}(E)$ due to the leads are evaluated by Eqs. (5) and (9). As these terms are independent of disorder configuration, these need only be evaluated once and then stored. With $\overline{\mathbf{\Sigma}}_{C}^{\text {ret, }}<$ initially set equal to zero, $\overline{\mathbf{G}}^{<}$and $\overline{\mathbf{G}}^{\text {ret }}$ are calculated. These $\overline{\mathbf{G}}$ 's are used to calculate the $\overline{\mathbf{\Sigma}}_{C}$ 's, via an appropriate approximation scheme. The updated $\overline{\mathbf{\Sigma}}_{C}$ 's are used to generate new $\overline{\mathbf{G}}$ 's via Eqs. (7) and (14), and the process is iterated until convergence is achieved. Finally, the current is evaluated with Eq. (3).

In Appendix A we show explicitly that the ladder approximation for the vertex function in the transmission formulation yields the same equations as the Keldysh approach within the self-consistent Born approximation, demonstrating the equivalence of the two methods for impurity scattering. Nevertheless the Keldysh approach seems to be conceptually easier as there is only one place within this formulation where an approximation is made; i.e., in the selfenergy. In contrast, with the transmission formalism, errors can occur if the vertex function does not fulfill the Ward identity, providing a pitfall to trap the uninitiated and unwary.

\section{APPLICATION TO A SUPERLATTICE STRUCTURE}

Let us consider the superlattice structure sketched schematically in Fig. 1. The superlattice consists of $N$ identical wells embedded in $N+1$ barriers. A bias $U$ is applied to the structure yielding constant potentials $U_{L}$ and $U_{R}=U_{L}+e U$ at the left and right contact, respectively. In order to perform calculations we now specify the basis states $\phi_{C, j}(\vec{r})$ and $\chi_{l \alpha}(\mathbf{r})$ for our superlattice structure. The lead index $l$ takes two different values $L$ and $R$, for the left and right contact region, respectively. For superlattices with a large cross section $A$ it is natural to use a basis of plane waves $e^{i \mathbf{k} \cdot \mathbf{r}} / \sqrt{A}$ for the transverse coordinates $(x, y)$ both in the lead regions and in the superlattice itself. Then the index $\alpha$ of the states in the leads is replaced by $\mathbf{k}$ and we have $E_{(L / R, \mathbf{k})}=E_{k}+U_{L / R}$, where $E_{k}=\hbar^{2} k^{2} / 2 m$.

\section{A. Wannier approximation for a superlattice}

Let us now consider the central region; i.e., the superlattice structure itself. In order to make a calculation tractable, we restrict ourselves to a subset of the basis functions of total Hilbert space, ignoring irrelevant high-energy states. With respect to the $z$ direction inside the superlattice we use a basis of Wannier functions $\Psi_{n}(z)(n=1, \ldots N)$ from the lowest miniband which are maximally localized in well $n{ }^{26}$ Such a basis has been successfully applied to superlattice transport. ${ }^{27}$ This approximation, which we call the Wannier approximation (WA), neglects higher minibands, and its validity is discussed in Appendix B. There we demonstrate that this approximation gives good results for the transmission probability provided that the miniband width is smaller than the energy of the center of the miniband and the energy range of interest is sufficiently below the levels corresponding to the higher miniband states. The states $\phi_{C, j}$ within the superlattice are, within the WA, given by products $\Psi_{n}(z) e^{i \mathbf{k} \cdot \mathbf{r}} / \sqrt{A}$, which can be labeled by $(n, \mathbf{k})$.

Within the superlattice, the Green function is determined by Eq. (4) which in the WA basis reads

$$
\begin{aligned}
\sum_{n^{\prime} \mathbf{k}^{\prime}} & {\left[\left(E-E^{a}-E_{k}-U_{n}\right) \delta_{\mathbf{k}, \mathbf{k}^{\prime}} \delta_{n, n^{\prime}}\right.} \\
& -H_{n \mathbf{k}, n^{\prime} \mathbf{k}^{\prime}}-T_{1} \delta_{\mathbf{k}, \mathbf{k}^{\prime}}\left(\delta_{n, n^{\prime}+1}+\delta_{n, n^{\prime}-1}\right) \\
& \left.-\sum_{l \alpha} \Sigma_{l \alpha ; n \mathbf{k}, n^{\prime} \mathbf{k}^{\prime}}^{\mathrm{ret}}(E)\right] G_{n^{\prime} \mathbf{k}^{\prime}, m \mathbf{k}_{1}}^{\mathrm{ret}}(E)=\delta_{\mathbf{k}, \mathbf{k}_{1}} \delta_{n, m} .
\end{aligned}
$$

Here, $U_{n}$ denotes the potential in the well $n$ (see Fig. 1) which is due to an external bias. (The mean-field potential induced by the carriers in the structure can be added as well.) $T_{1}$ is the coupling between the wells and $E^{a}$ is the level energy of the Wannier state relative to the bottom of the well. For a given structure, we calculate $T_{1}$ and $E^{a}$ as follows. We consider first an infinite superlattice of the same composition. The eigenstates in the infinite superlattice are Bloch functions with the miniband dispersion $E^{a}(q) . E^{a}$ is then identified as the center of the miniband $d /(2 \pi) \int d q E^{a}(q)$ and $T_{1}=d /(2 \pi) \int d q E^{a}(q) \cos (q d)$, where $d$ is the period of the superlattice, ${ }^{27}$ i.e., $\left|T_{1}\right|$ is about one-fourth of the miniband width. Finally, $H_{n \mathbf{k}, n^{\prime} \mathbf{k}^{\prime}}$ is the disorder scattering matrix element.

If we average over disorder configurations the translational invariance in the $(x, y)$ plane is restored, and consequently, all impurity-averaged quantities are diagonal in $\mathbf{k}$ parallel to the $(x, y)$ plane. Therefore we are able to use the 
notation $\bar{G}_{n \mathbf{k}, m \mathbf{k}}(E) \equiv \bar{G}_{n m}(\mathbf{k}, E)$ and matrices $\mathbf{G}(\mathbf{k}, E)$ and $\boldsymbol{\Sigma}(\mathbf{k}, E)$ have the components $G_{n m}(\mathbf{k}, E)$ and $\Sigma_{n m}(\mathbf{k}, E)$, respectively.

\section{B. Estimating the coupling and wide-band limit}

The coupling with the mode $\mathbf{k}$ in the left contact yields, from Eq. (5), the self-energy

$$
\begin{aligned}
\sum_{L \mathbf{k} ; n \mathbf{k}_{1}, n^{\prime} \mathbf{k}_{2}}^{\mathrm{ret}}(E) \\
=\delta_{n, 1} \delta_{n^{\prime}, 1} \delta_{\mathbf{k}_{1}, \mathbf{k}} \delta_{\mathbf{k}_{2}, \mathbf{k}} \\
\quad \times \frac{1}{2 \pi} \int_{0}^{\infty} d E_{q} \frac{2 L_{L}\left|V_{q}\right|^{2}}{\hbar \mathbf{v}_{q}} \frac{1}{E-E_{q}-E_{\mathbf{k}}-U_{L}+i 0^{+}},
\end{aligned}
$$

where $V_{q}=\left\langle\varphi_{q}^{L}(z)\left|\hat{H}_{L C}\right| \Psi_{1}(z)\right\rangle$ is the z-dependent part of the matrix element for the coupling to the leads. Here we neglect the coupling to the inner wells $(n \neq 1)$, which should be small. The right contact gives the same term except with replacements $\delta_{n, 1} \rightarrow \delta_{n, N}$ and $U_{L}, L_{L} \rightarrow U_{R}, L_{R}$.

If the transmission function is strongly determined by resonances, only a small energy range of $E \approx E^{a}+U_{L}+E_{k}$ contributes to the transmission. In this range we neglect the $q$ dependence of the coupling and extend the lower limit of the integration in Eq. (16) to $-\infty$. Then we obtain for the left lead

$$
\sum_{L \mathbf{k} ; n \mathbf{k}_{1}, n^{\prime}, \mathbf{k}_{2}}^{\mathrm{ret}}(E)=\delta_{n, 1} \delta_{n^{\prime}, 1} \delta_{\mathbf{k}_{1}, \mathbf{k}} \delta_{\mathbf{k}_{2}, \mathbf{k}} \frac{-i}{2} \Gamma_{L}
$$

with

$$
\Gamma_{L}=\frac{2 L_{L}\left|V_{q\left(E^{a}\right)}\right|^{2}}{\hbar \mathrm{v}_{q\left(E^{a}\right)}} .
$$

This approximation is often referred to as wide-band limit. Note that this limit becomes problematic if the voltage drop across the first barrier becomes large, as this changes the relevant values of $E$ and it cannot be regarded as constant (see also Appendix B).

Now we want to estimate the value of $\left|V_{q}\right|^{2}$. For $E_{q}$ $\approx E^{a}$ the wave function $\varphi_{q}^{L}(z)$ in the left lead behaves like the Wannier function $\Psi_{0}(z)=\Psi_{1}(z+d)$ which is localized in a fictitious additional well on the left side of the structure. Now $\varphi_{q}^{L}(z)$ is normalized to $L_{L}$ while the spatial extension of the Wannier function is given by $w_{\text {eff }}$, which should be slightly larger than the well width, as the function penetrates into the barriers. Therefore we may set $\varphi_{q}^{L}(z)$ $\sim \sqrt{w_{\text {eff }} / L_{L}} \Psi_{0}(z)$. Then we can estimate the matrix element

$$
\left\langle\varphi_{q}^{L}|H| \Psi_{1}\right\rangle \approx \sqrt{\frac{w_{\mathrm{eff}}}{L_{L}}}\left\langle\Psi_{0}|H| \Psi_{1}\right\rangle=\sqrt{\frac{w_{\mathrm{eff}}}{L_{L}}} T_{1},
$$

yielding

$$
\Gamma_{L} \approx \frac{2 w_{\mathrm{eff}} T_{1}^{2}}{\hbar \mathrm{v}_{q\left(E^{a}\right)}}
$$

For the right contact, $\Gamma_{R}$ is given by the same value.

\section{Interface roughness}

For ideal structures the potential $H_{n \mathbf{k}, n^{\prime} \mathbf{k}^{\prime}}$ in Eq. (15) is zero due to the translational invariance within the $(x, y)$ plane. However, interface fluctuations leading to well width fluctuations $\xi_{n}(\mathbf{r})$ in real samples break this translational invariance. If interwell scattering and well-width correlations between different wells can be neglected, the averaged square of the scattering matrix element is given by ${ }^{28,29}$

$$
\left\langle\left|H_{n \mathbf{k}+\mathbf{p}, n^{\prime} \mathbf{k}}\right|^{2}\right\rangle=\frac{K^{2}}{A} S(\mathbf{p}) \delta_{n, n^{\prime}},
$$

where $K$ is equal to the change of energy $d E^{a} / d w$ per well width fluctuation ${ }^{30}$ and $S(\mathbf{p})$ is the Fourier transformation of the well-width correlation function $\left\langle\xi_{n}(\mathbf{r}) \xi_{n}\left(\mathbf{r}^{\prime}\right)\right\rangle=f(\mathbf{r}$ $\left.-\mathbf{r}^{\prime}\right)$ which is assumed to be independent of the well index. The theory can be extended to accommodate interwell scattering and well-width correlations between different wells (which may result from a repetition of the microscopic interface structure over several superlattice periods) by the inclusion of the appropriate correlation functions $\left\langle H_{n_{1} \mathbf{k}-\mathbf{p}, n_{1}^{\prime} \mathbf{k}} H_{n_{2} \mathbf{k}+\mathbf{p}, n_{2}^{\prime} \mathbf{k}}\right\rangle$. We use an isotropic exponential distribution $f(r)=\eta^{2} \exp (-r / \lambda)$ yielding

$$
S(\mathbf{p})=\eta^{2} \lambda^{2} \frac{2 \pi}{\left[1+(p \lambda)^{2}\right]^{3 / 2}},
$$

where $\eta$ denotes the standard deviation and $\lambda$ the in-plane correlation length of the well-width fluctuation. It is straightforward to implement more sophisticated distribution functions, which might be obtained from Monte Carlo simulations of the growth conditions (see, e.g., Ref. 31) or x-ray characterizations of the superlattice structure (see, e.g., Ref. 32). Within the self-consistent Born approximation we obtain the self energy $\overline{\boldsymbol{\Sigma}}_{C}$,

$$
\bar{\Sigma}_{C ; n n}^{</ \text {ret }}(\mathbf{k}, E)=\sum_{\mathbf{k}^{\prime}}\left\langle\left|H_{\mathbf{k}^{\prime}, \mathbf{k}}\right|^{2}\right\rangle \bar{G}_{n n}^{</ \mathrm{ret}}\left(\mathbf{k}^{\prime}, E\right)
$$

which provides the functional needed in the procedure sketched in Sec. II C.

\section{RESULTS}

Let us consider the transmission of ballistic electrons through the superlattices considered in recent experiments by Rauch et al. ${ }^{8}$ The structure consists of $N$ wells of $6.5-\mathrm{nm}$ GaAs and $N+1$ barriers of $2.5-\mathrm{nm} \mathrm{Al}_{0.3} \mathrm{Ga}_{0.7} \mathrm{As}$. We obtain the band parameters $E^{a}=54.5 \mathrm{meV}, T_{1}=-5.84 \mathrm{meV}, K$ $=13.25 \mathrm{meV} / \mathrm{nm}$ and use $w_{\text {eff }}=10.7 \mathrm{~nm}$, where we obtained the best agreement with "exact" calculations; see Appendix B. This value is somewhat larger than the well width in good agreement with the discussion in Sec. III B. We assume thickness fluctuations of half a monolayer $\eta=0.14 \mathrm{~nm}$ around the nominal value and a correlation length $\lambda$ $=5 \mathrm{~nm}$, unless otherwise stated.

Motivated by the relatively sharp electron distribution injected into the structure, we assume that the electrons occupy the mode $\mathbf{k}=0$ of the left contact at an energy $E=E_{\text {in }}$; i.e., 

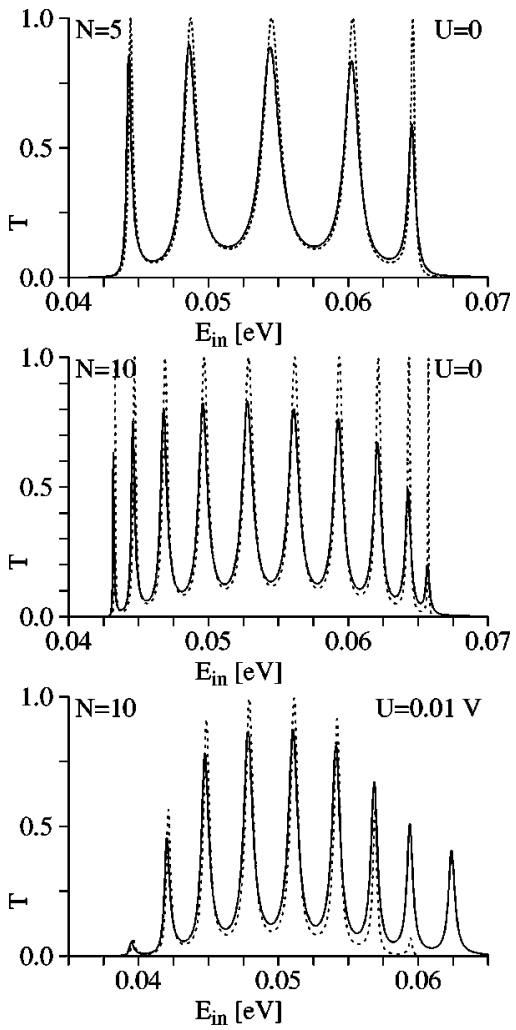

FIG. 2. Transmission through superlattices for different lengths and biases: The full line depicts the transmission calculated from Eq. (24) for a superlattice structure with interface roughness. The dotted line gives the transmission for an ideal superlattice without scattering.

we have $f_{L \mathbf{k}}(E)=\delta_{\mathbf{k}, 0} \delta\left(E-E_{i n}\right)$ and $f_{R \mathbf{k}}(E)=0$. The total current through the right contact is then given by

$$
J_{R}=\sum_{\mathbf{k}} J_{R \mathbf{k}}=-\frac{e}{\pi \hbar} \sum_{\mathbf{k}} \int d E \operatorname{Tr}\left\{-i \boldsymbol{\Gamma}_{R \mathbf{k}}(E) \mathbf{G}^{<}(E)\right\} .
$$

This can be expressed via Eq. (12) by

$$
J_{R}=-\frac{e}{\pi \hbar} \sum_{\mathbf{k}} T_{(R, \mathbf{k}) \leftarrow(L, \mathbf{0})}\left(E_{\mathrm{in}}\right) .
$$

For illustrative purpose we calculate the effective transmission $T\left(E_{\mathrm{in}}\right)=-J_{R} \pi \hbar / e$ in the following. Regarding the applied bias we assume a homogeneous electric field $F$ inside the superlattice and set $U_{L}=0, U_{n}=-(n-1 / 2) e F d$ $-e F b / 2$ and $U_{R}=-N e F d-e F b=e U$ where $b$ is the barrier width. In the experiments considered, there is no charge accumulation inside the structure as there is on average less than one electron inside the structure at a given time. If necessary such effects can be easily taken into account by solving the Poisson equation for the electron density given by

$$
N_{n}=\frac{-i}{2 \pi A} \sum_{\mathbf{k}} \int d E \bar{G}_{n n}^{<}(\mathbf{k}, E) .
$$

In Fig. 2 we show the effective transmission with and without scattering. In both cases we find a series of peaks, equal to the number of quantum wells, which reflect the eigenstates of the superlattice structure. For $U=0$ the peak
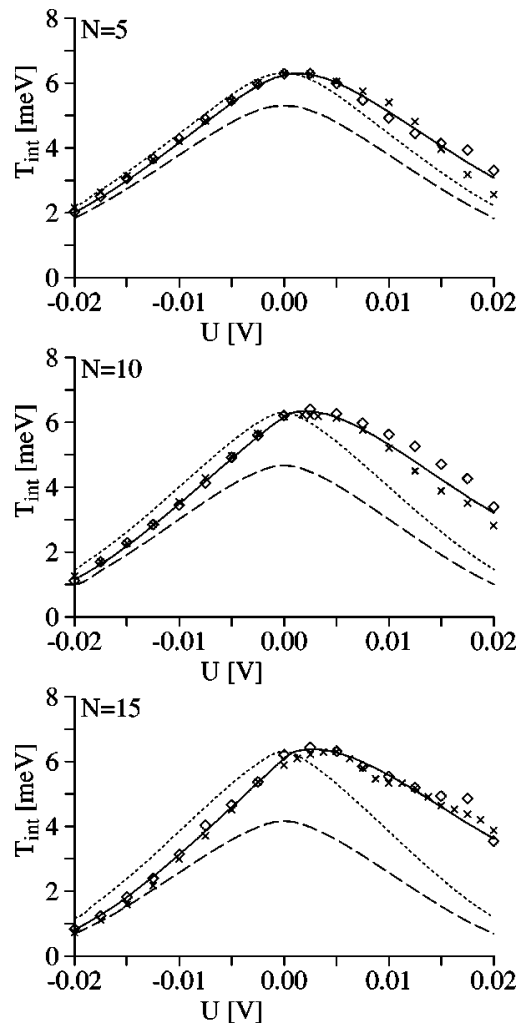

FIG. 3. Integrated transmission from Eq. (27) through superlattices for different lengths: The full line depicts the transmission calculated from Eq. (24) for a superlattice structure with interface roughness. The dashed line denotes the part of transmission without scattering. The dotted line gives the result for an ideal superlattice without scattering for comparison. The crosses and diamonds give the results calculated for the same structure within the model of Ref. 11 for two different realizations of the interface roughness.

maxima reach the value 1 for the ideal superlattice. The broadening of these peaks results from the coupling to the leads and is of the order $\left(\Gamma_{R}+\Gamma_{L}\right) / N$. In contrast the maxima are lower and the widths are wider for the calculation including scattering. These effects becomes more pronounced with increasing superlattice length as the broadening due to scattering dominates with respect to the lead induced broadening.

An important quantity is the integrated transmission for a given potential drop $U$

$$
T_{\text {int }}(U)=\int d E_{\mathrm{in}} T\left(E_{\mathrm{in}} ; U\right),
$$

where the integration is extended over the whole energy range of the band. This quantity was measured in Refs. 8 and 10. Results are shown in Fig. 3. Let us compare the result of the calculations with (full line) and without roughness (dotted line) first. Without interface roughness, the function $T_{\text {int }}(U)$ is always symmetric with respect to $U$. This can be understood from the symmetry property of the transmission matrix $T_{l \alpha \leftarrow l^{\prime} \beta}(E)=T_{l^{\prime} \beta \leftarrow l \alpha}(E)$ (see, e.g., Ref. 18). For an ideal structure, $\mathbf{k}$ is conserved within the structure and we find according to Eq. (25) 


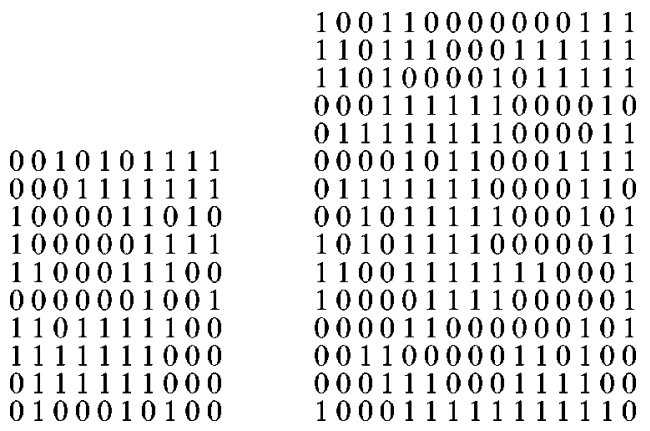

FIG. 4. Two different realizations of interface structure used within the model of Ref. 11. The left one is used for the crosses and the right one for the diamonds in Fig. 3. Here we show the distribution of the second well, the other distributions have identical statistical properties. The spatial discretization is assumed to be 5 nm. Both distribution give a spatial correlation $\langle(\xi(\mathbf{r})$ $\left.-\langle\xi(\mathbf{r})\rangle)\left(\xi\left(\mathbf{r}^{\prime}\right)-\left\langle\xi\left(\mathbf{r}^{\prime}\right)\right\rangle\right)\right\rangle \approx 0.5^{2} \exp \left(-\left|\mathbf{r}-\mathbf{r}^{\prime}\right| / 5 \mathrm{~nm}\right)$ within the next two neighbors. $[\xi(\mathbf{r})$ denotes the local fluctuation.]

$$
\begin{aligned}
T_{\mathrm{int}}(U) & =\int d E_{\mathrm{in}} \sum_{\mathbf{k}} T_{(R, \mathbf{k}) \leftarrow(L, \mathbf{0})}\left(E_{\mathrm{in}} ; U\right) \\
& =\int d E_{\mathrm{in}} T_{(R, \mathbf{0}) \leftarrow(L, \mathbf{0})}\left(E_{\mathrm{in}} ; U\right) \\
& =\int d E_{\mathrm{in}} T_{(L, \mathbf{0}) \leftarrow(R, \mathbf{0})}\left(E_{\mathrm{in}} ; U\right) .
\end{aligned}
$$

Now $T_{(L, \mathbf{0}) \leftarrow(R, \mathbf{0})}\left(E_{\text {in }} ; U\right)=T_{(R, \mathbf{0}) \leftarrow(L, \mathbf{0})}\left(E_{\text {in }}+e U ;-U\right)$ due to the symmetry of the structure and so we find $T_{\text {int }}(U)$ $=T_{\text {int }}(-U)$.

This argument does not hold for a superlattice with interface roughness as the scattering is able to transfer electrons from state $\mathbf{k}=\mathbf{0}$, where they are injected to a finite value of k. In this case kinetic energy $E_{k}$ is transferred to the $(x, y)$ direction and the electrons leave the superlattice with a lower $z$ component of the energy $E_{q}$. This opens up new channels for new processes if $U>0$; see also the discussion in Ref. 11. Therefore the function $T_{i n t}(U)$ is asymmetric with respect to the bias $U$ as can be clearly seen in Fig. 3 (full line). These findings are in excellent agreement with recent measurements. ${ }^{10}$

In Fig. 3 we have also shown the transmission due to electrons traversing the superlattice without scattering (dashed line). This curve is obtained by neglecting the term $\Sigma_{C}^{<}(E)$ in Eq. (8). It can be clearly seen that this curve is symmetric with respect to the bias and its magnitude is decreasing with increasing sample length.

An alternative way of calculating the transmission has been performed in Refs. 11. There the Green functions were calculated for a fixed interface potential following Ref. 16. For practical reasons the size of the samples is relatively small. The diamonds and crosses refer to two different random interface potentials as shown in Fig. 4 which both have approximately the same statistical features. The data obtained for the transmission are not smooth for $U>0$ and exhibit differences between each other. This indicates that significantly larger areas than $10 \times 10$ or $15 \times 15$ grid points must be used for reliable calculations utilizing this method, which is not practicable. In contrast the method using impu-
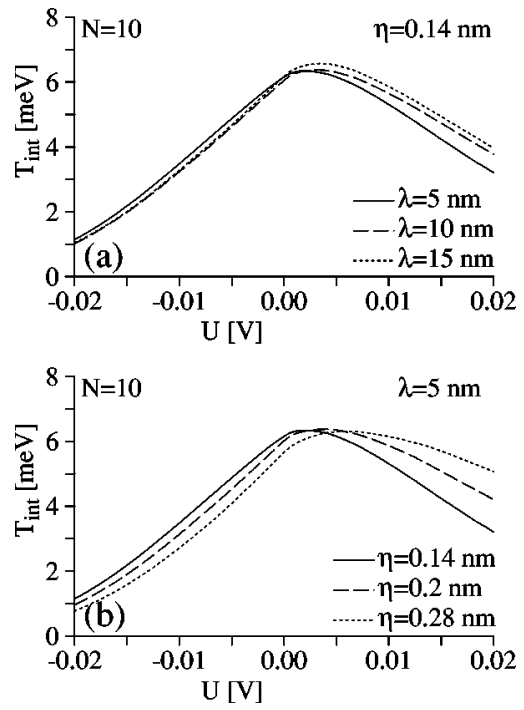

FIG. 5. Integrated transmission through superlattices with different correlation lengths (a) and different heights (b) of the interface roughness.

rity averaging presented here gives a smooth behavior which, in effect, averages the scattered data points obtained from the previous calculations.

In Fig. 5(a) we have shown the integrated transmission for different values of the correlation length for the roughness distributions. In the range considered we find that the asymmetry increases with the correlation length of the interface roughness. This indicates that larger islands lead to an enhancement of scattering even if the average coverage is identical. The reason is that scattering events with low momentum transfer is enhanced. Such scattering events dominate the transport characteristics of the superlattice due to the energy scales involved in the system. Figure 5(b) shows the increase of the asymmetry with the fluctuation height. The strong dependence allows for an estimation of the interface quality by analyzing the experimental transmission data.

\section{SUMMARY AND CONCLUSIONS}

We have presented a formalism to calculate the transmission of electrons through a finite superlattice in the presence of scattering processes. Due to impurity averaging the results are applicable to samples with large cross sections. We have also shown that reasonable results can be obtained by restricting the calculation to a basis of Wannier functions. Within this Wannier approximation all couplings are well defined and can be easily calculated from the superlattice parameters, with the only slight ambiguity being the effective normalization width $w_{\text {eff }}$, which is typically a few nanometers larger than the well width.

Although we have only presented results for interface roughness scattering, the formalism is easily applicable to other elastic scattering processes, such as impurity scattering, as well. With regard to inelastic phonon scattering, the formalism holds as well if Langreth rules ${ }^{33,22}$ are taken into account, which provide the more complicated functionals for the retarded and lesser self-energies, see also Ref. 34. Nevertheless, one encounters the problem that the Green functions at different energies couple to each other. Therefore the 
set of equations which has to be solved self-consistently becomes significantly larger. The inclusion of electron-electron interaction within the mean-field model is straightforward.

Our results show that interface roughness gives an enhancement of the electron transmission for positive biases applied to the superlattice. The shape of the integrated transmissions depends strongly on the distribution of the well width fluctuations and allows us to study interface roughness in semiconductor heterostructures. This provides a complementary approach to the usual method of characterization by luminescence spectra.

\section{ACKNOWLEDGMENTS}

Helpful discussions with S. Bose, W. Boxleitner, F. Elsholz, E. Gornik, A.-P. Jauho, G. Kießlich, and C. Rauch are acknowledged. This work has been supported by the Deutsche Forschungsgemeinschaft in the framework of SFB 296.

\section{APPENDIX A: IMPURITY AVERAGING}

In this appendix we describe the procedure of disorder averaging for the superlattice structure discussed in Sec. III. The cross section $A$ of typical superlattices is large enough that the transmission from $L$ to $R$ is given by the configurational average of impurities. For the sake of transparency we assume that the scattering matrix element is diagonal in the well index and that the impurities are uncorrelated between different wells. The inclusion of both effects is straightforward and the identities derived below hold in a similar way if $\left|U\left(\mathbf{k}-\mathbf{k}^{\prime}\right)\right|^{2}$ is generalized to $\left\langle U_{n_{1} n_{1}^{\prime}}\left(\mathbf{k}-\mathbf{k}^{\prime}\right) U_{n_{2} n_{2}^{\prime}}\left(\mathbf{k}^{\prime}\right.\right.$ $-\mathbf{k})\rangle$.

\section{Transmission formulation}

The formalism described here is similar to one used in Ref. 35, except that that work was concerned with a resonant tunneling device (i.e., one well in the structure), and the scattering was calculated only to lowest order. Hence only single scattering events were included, whereas the formalism described here takes multiscattering events into account. This is important for superlattices, as it is unlikely for an electron to pass through a relatively long structure with only one collision.

From Eqs. (13) and (16), the averaged transmission matrix can be written

$$
\bar{T}_{R \mathbf{k} \leftarrow l_{1} \mathbf{k}_{1}}(E)=\Gamma_{R, \mathbf{k}}(E) \Pi\left(N \mathbf{k}, l_{1} \mathbf{k}_{1} ; E\right)
$$

with

$$
\Pi\left(n \mathbf{k}, l_{1} \mathbf{k}_{1} ; E\right)=\overline{G_{n \mathbf{k}, n_{1}\left(l_{1}\right) \mathbf{k}_{1}}^{\mathrm{ret}}(E) \Gamma_{l_{1} \mathbf{k}_{1}}(E) G_{n_{1}\left(l_{1}\right) \mathbf{k}_{1}, n \mathbf{k}}^{\mathrm{adv}}(E)},
$$

where the overline denotes impurity averaging, $l_{1}=L$ or $R$, and we define $n_{1}(L)=1$ and $n_{1}(R)=N$. This problem is analogous to the well-known case of impurity scattering in bulk material (see, e.g., Ref. 25). The impurity averaging introduces a self-energy $\Sigma^{\mathrm{ret} / \mathrm{adv}}(\mathbf{k}, E)$ to the Green functions and, as in the bulk case, vertex corrections due to impurity potential correlations between $G^{\text {ret }}$ and $G^{\text {adv }}$. Within the self- consistent Born approximation, in addition to the impurity contribution to the self-energy, one must keep the ladder diagrams in the vertex. ${ }^{25}$

We assume that every well has the same uncorrelated randomly distributed concentration of impurities with areal density $n_{\text {imp }}$, and each impurity has a Fourier transformed potential $U(q)$. Within the self-consistent Born approximation the retarded self-energy is diagonal within the well coordinates $n, m$ (i.e., $\bar{\Sigma}_{C, n m}^{\text {ret }}=\bar{\Sigma}_{C, n}^{\text {ret }} \delta_{n m}$ ), and is given by

$$
\bar{\Sigma}_{C, n}^{\mathrm{ret}}(\mathbf{k}, E)=n_{\text {imp }} \int \frac{d \mathbf{k}^{\prime}}{(2 \pi)^{2}}\left|U\left(\mathbf{k}-\mathbf{k}^{\prime}\right)\right|^{2} \bar{G}_{n n}^{\mathrm{ret}}\left(\mathbf{k}^{\prime}, E\right) .
$$

Higher-order approximations have been used in Ref. 36 for the resonant tunneling diode. The impurity-averaged Green function is obtained from Eq. (4), and is explicitly given by

$$
\begin{aligned}
{\left[\bar{G}^{\mathrm{ret}^{-1}}(\mathbf{k}, E)\right]_{n m}=} & {\left[G_{0}^{\mathrm{ret}^{-1}}(\mathbf{k}, E)\right]_{n m}-\delta_{n m}\left[\Sigma_{C, n}^{\mathrm{ret}}(\mathbf{k}, E)\right.} \\
& \left.+\Sigma_{L}^{\mathrm{ret}}(\mathbf{k}, E) \delta_{n 1}+\Sigma_{R}^{\mathrm{ret}}(\mathbf{k}, E) \delta_{n N}\right],
\end{aligned}
$$

where $n, m=1, \ldots, N$ are the well coordinates. The ladder approximation for $\Pi\left(n \mathbf{k}, l_{1} \mathbf{k}_{1} ; E\right)$ yields

$$
\begin{aligned}
\Pi\left(n \mathbf{k}, l_{1} \mathbf{k}_{1} ; E\right)= & \bar{G}_{n n_{1}\left(l_{1}\right)}^{\mathrm{ret}}(\mathbf{k}, E) \Gamma_{l_{1} \mathbf{k}}(E) \bar{G}_{n_{1}\left(l_{1}\right) n}^{\mathrm{adv}}(\mathbf{k}, E) \delta_{\mathbf{k k}_{1}} \\
& +\sum_{m=1}^{N} n_{\mathrm{imp}} \int \frac{d \mathbf{k}^{\prime}}{(2 \pi)^{2}}\left|U\left(\mathbf{k}-\mathbf{k}^{\prime}\right)\right|^{2} \\
& \times \bar{G}_{n m}^{\mathrm{ret}}(\mathbf{k}, E) \Pi\left(m \mathbf{k}^{\prime}, l_{1} \mathbf{k}_{1} ; E\right) \bar{G}_{m n}^{\mathrm{adv}}(\mathbf{k}, E)
\end{aligned}
$$

This equation can be iterated to yield $\Pi\left(n \mathbf{k}, l_{1} \mathbf{k}_{1} ; E\right)$. Note here that $l_{1} \mathbf{k}_{1}$ acts merely as a parameter. As we assume that particles are injected from the left side into the superlattice with zero transverse momentum, we need only calculate the case $l_{1}=L$ and $\mathbf{k}_{1}=0$.

\section{Keldysh formulation}

For comparison, we give below the equations which arise from the Keldysh formulation of this problem, within the same approximations described above. The retarded Green function is determined by Eqs. (A3) and (A4), as in the previous subsection. In addition we have

$$
\Sigma_{C, n}^{<}(\mathbf{k}, E)=n_{\text {imp }} \int \frac{d \mathbf{k}^{\prime}}{(2 \pi)^{2}}\left|U\left(\mathbf{k}-\mathbf{k}^{\prime}\right)\right|^{2} \bar{G}_{n n}^{<}\left(\mathbf{k}^{\prime}, E\right) .
$$

Together with Eqs. (7) and (8) we obtain

$$
\begin{aligned}
\Sigma_{n}^{<}(\mathbf{k}, E)= & i \sum_{l} f_{l \mathbf{k}}(E) \Gamma_{l \mathbf{k}}(E) \delta_{n n_{1}(l)} \\
& +n_{\mathrm{imp}} \sum_{m} \int \frac{d \mathbf{k}^{\prime}}{(2 \pi)^{2}}\left|U\left(\mathbf{k}-\mathbf{k}^{\prime}\right)\right|^{2} \\
& \times \bar{G}_{n m}^{\mathrm{ret}}\left(\mathbf{k}^{\prime}, E\right) \Sigma_{m}^{<}\left(\mathbf{k}^{\prime}, E\right) \bar{G}_{m n}^{\mathrm{adv}}\left(\mathbf{k}^{\prime}, E\right) .
\end{aligned}
$$


The numerical expense required to find the self-consistent solutions for $\mathbf{\Sigma}^{<}(\mathbf{k}, E)$ and to solve for Eq. (A5) are the same. The final evaluation of $\bar{G}_{n n^{\prime}}^{<}(\mathbf{k}, E)$ $=\Sigma_{m} \bar{G}_{n m}^{\mathrm{ret}}(\mathbf{k}, E) \Sigma_{m}^{<}(\mathbf{k}, E) \bar{G}_{m n^{\prime}}^{\mathrm{adv}}(\mathbf{k}, E)$ is straightforward and we can identify

$$
i \sum_{l_{1} \mathbf{k}_{\mathbf{1}}} \Pi\left(n \mathbf{k}, l_{1} \mathbf{k}_{1} ; E\right) f_{l_{1} \mathbf{k}_{\mathbf{1}}}(E)=\bar{G}_{n n}^{<}(\mathbf{k}, E),
$$

as both sides are determined by an identical set of equations. Similarly, one can show

$$
-i \sum_{l_{1} \mathbf{k}_{1}} \Pi\left(n \mathbf{k}, l_{1} \mathbf{k}_{1} ; E\right)=\bar{G}_{n n}^{\mathrm{ret}}(\mathbf{k}, E)-\bar{G}_{n n}^{\mathrm{adv}}(\mathbf{k}, E)
$$

by using the identity $\bar{G}_{n n^{\prime}}^{\mathrm{ret}}(\mathbf{k}, E)-\bar{G}_{n n^{\prime}}^{\mathrm{adv}}(\mathbf{k}, E)=$ $-i \Sigma_{m} \bar{G}_{n m}^{\mathrm{ret}}(\mathbf{k}, E) \Gamma_{m}(\mathbf{k}, E) \bar{G}_{m n^{\prime}}^{\mathrm{adv}}(\mathbf{k}, E) \quad$ where $\quad \Gamma_{n}(\mathbf{k}, E)$ $=i\left[\sum_{n}^{\mathrm{ret}}(\mathbf{k}, E)-\Sigma_{n}^{\mathrm{adv}}(\mathbf{k}, E)\right]$ satisfies an equation very much like Eq. (A7). The identities Eqs. (A8) and (A9) show that the Landauer-Büttiker expression Eq. (12) with the averaged transmission matrix Eq. (A1) is identical to the Keldysh formulation result given by Eq. (3). This explicitly demonstrates the equivalence of the transmission and the Keldysh approaches within the self-consistent Born approximation of the scattering.

\section{APPENDIX B: THE ACCURACY OF THE WANNIER AND WIDE-BAND APPROXIMATION}

In order to check the accuracy of both the Wannier approximation and the wide-band limit, we will compare our results with a different approach. Calculations in real space have been performed in Refs. 16-18 using a fine spatial discretization of length $a$. In the limit $a \rightarrow 0$, these calculations in principle yield exact results. Unfortunately, these approaches generate huge matrices, so that we restrict ourselves to a one-dimensional structure and neglect the $(x, y)$ direction. This refers to an ideal superlattice, where the $z$ and $(x, y)$ directions decouple. Numbering the discretization points with indices $i$ the total Hamiltonian is then given by

$$
H_{i j}=V_{i} \delta_{i, j}+\tau_{i j}
$$

with

$$
\begin{gathered}
\tau_{i j}=-\frac{\hbar^{2}}{4 a^{2}}\left(\frac{1}{m_{i}^{*}}+\frac{1}{m_{j}^{*}}\right) \quad \text { for nearest neighbors, } \\
\tau_{i i}=\frac{\hbar^{2}}{a^{2} m_{i}^{*}},
\end{gathered}
$$

where a position dependent effective mass $m_{i}^{*}$ has been included following Ref. 16. Now we assume that the sample is translationally invariant in the $z$ direction for discretization points $i<0$ and $i>M$ and the coupling term in these regions is $\tau_{i, i \pm 1}=t<0$. Then we may define the region $0 \leqslant i \leqslant M$ as the structure and the regions $i<0$ and $i>M$ as leads within the formalism given above. The solutions for $j<0$ are plane waves $\sin \left[q_{L}(E) j a\right]$ and we have

$$
E=U_{L}+2 t\left[\cos \left(q_{L} a\right)-1\right] .
$$

For $U_{L}>E$ this gives an imaginary $q$ corresponding to a nonpropagating mode. [For a practical calculation $|t|$ should be larger than $\left|E-U_{L}\right|$; otherwise Eq. (B4) does not represent the effective mass parabola for the leads.] Cutting of the leads gives a self-energy (see Chap. 3.5 of Ref. 18)

$$
\Sigma_{00}^{\mathrm{ret}}(E)=\left\{\begin{array}{ccc}
t \exp \left(i\left|q_{L}(E) a\right|\right) & \text { for } & E>U_{L} \\
t \exp \left(-\left|\kappa_{L}(E) a\right|\right) & \text { for } & E<U_{L}
\end{array},\right.
$$

where $\kappa_{L}(E)$ is defined by $E=U_{L}+2 t\left[\cosh \kappa_{L}(E) a-1\right]$ for $E<U_{L}$. Similar relations hold for $j=M$ with the mode from the right contact $R$. Note that this expression is only valid if the coupling from the lead to the central region is given by the same element $t$ as used in the discretization of the lead itself. The self-energy is added to the potential and we obtain the matrix equation
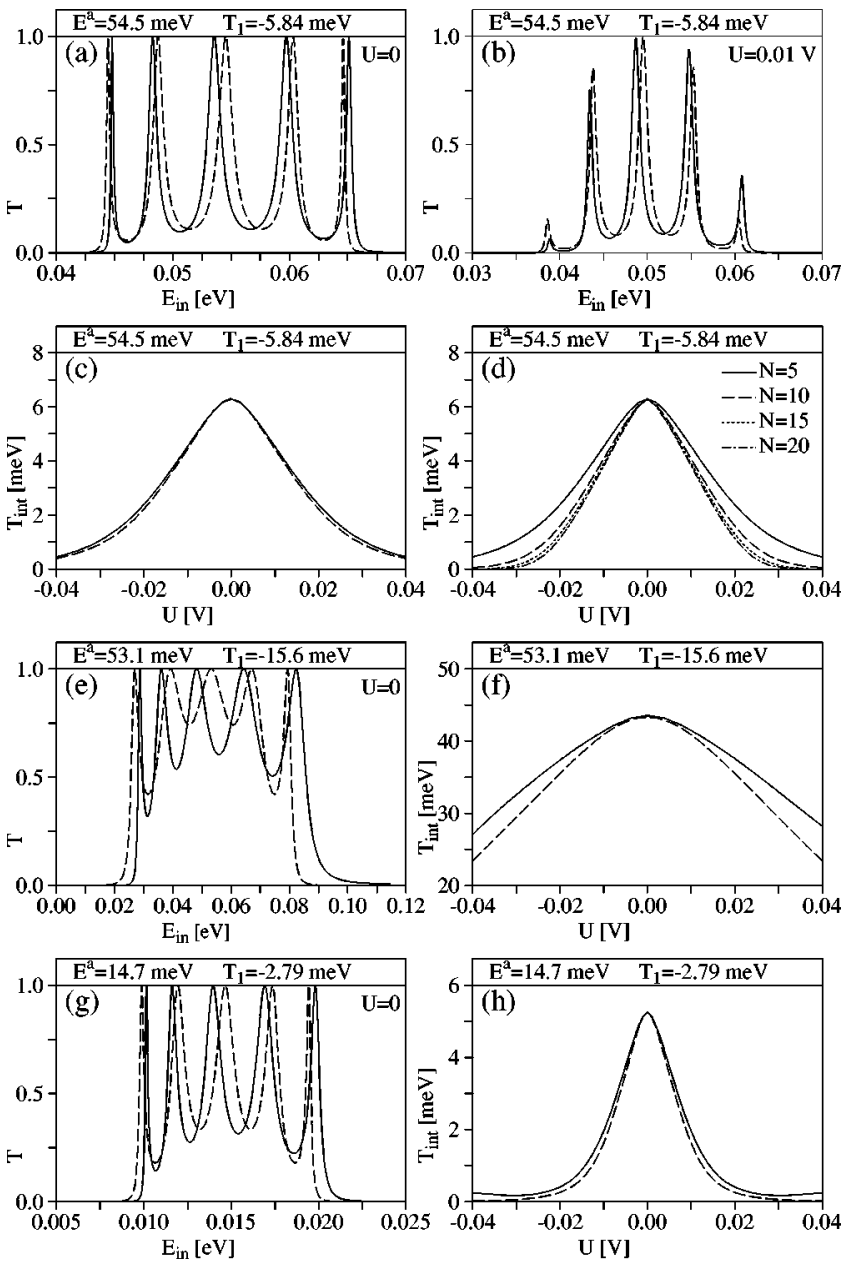

FIG. 6. Transmission through ideal superlattices $(N=5)$ without interface roughness. (a)-(c) and (e)-(h): Comparison between the "exact" model with fine discretization (full line) and the Wannier approximation (dashed line) for superlattices with five wells. (d) Comparison of the integrated transmission for different superlattice lengths using fine discretization. The superlattice has GaAs wells with widths of $6.5 \mathrm{~nm}(\mathrm{a})-(\mathrm{f})$ and $15 \mathrm{~nm}(\mathrm{~g})$ and (h), and $\mathrm{Al}_{0.3} \mathrm{Ga}_{0.7} \mathrm{As}$ barriers with widths of $2.5 \mathrm{~nm}$ (a)-(d) and $1 \mathrm{~nm}$ (e)(h). The calculated parameters for the center of the miniband $E^{a}$ and the coupling $T_{1}$ (about one-fourth of the miniband width) are given on top of each graph. 


$$
\left[E \delta_{i, j^{\prime}}-H_{i j^{\prime}}-\Sigma_{i j^{\prime}}^{\mathrm{ret}}\right] G_{j^{\prime} j}^{\mathrm{ret}}=\delta_{i, j}
$$

which can be inverted to evaluate the Green function. Finally the transmission is given by the Fisher-Lee relation, ${ }^{37}$ see also Chap. 3.4 of Ref. 18:

$$
T_{R, L}=4 t^{2} \sin \left|q_{L}(E) a\right| \sin \left|q_{R}(E) a\right|\left|G_{M, 0}^{\mathrm{ret}}(E)\right|^{2},
$$

which can be inserted in Eq. (12).

For comparison we consider a superlattice with $N=5$ wells of $6.5 \mathrm{~nm} \mathrm{GaAs}$ and 6 barriers of $2.5 \mathrm{~nm} \mathrm{Al} \mathrm{Al}_{0.3} \mathrm{Ga}_{0.7} \mathrm{As}$. Then we obtain the band parameters $E^{a}=54.5 \mathrm{meV}$ and $T_{1}$ $=-5.84 \mathrm{meV}$. The transmission is shown as a function of the injection energy $E_{\text {in }}$ for two different voltages in Figs. $6(\mathrm{a})$ and (b). Here we use $w_{\text {eff }}=10.7 \mathrm{~nm}$, where we obtained the best agreement. This value is somewhat larger than the well width in good agreement with the discussion in Sec. III B. We see that the transmission function contains five separate peaks which are related to the five states in the superlattice. The agreement between the approaches is quite good. If a bias is applied, the WA gives too high (low) transmissions for low (high) energies. The reason is the fact that the transmission through a barrier increases with energy, which is neglected in the WA. Preliminary results indicate that the agreement can be improved significantly, if nextnearest-neighbor couplings are included both in Eqs. (15) and (16). [In this case matrix elements like $\Sigma_{L \mathbf{k} ; 1 \mathbf{k}, 2 \mathbf{k}}^{\mathrm{ret}}(E)$ and $\sum_{L \mathbf{k} ; 2 \mathbf{k}, 2 \mathbf{k}}^{\mathrm{ret}}(E)$ have to be considered as well.]

The results for the integrated transmission $T_{\mathrm{int}}(U)$ are shown in Fig. 6(c). We find, that the WA gives good agreement with the discrete model for the integrated transmission. The agreement becomes even better if a larger barrier width is used (not shown here). In Fig. 6(d) we examine the length dependence of the integrated transmission calculated within the discrete model. We find that the function $T_{\text {int }}(U)$ be- comes almost independent of $N$ for large $N$. The results from the WA are almost indistinguishable and not shown here. Note, that $T_{i n t}(U)$ is a symmetric function with respect to the bias, which can be shown analytically using the symmetry properties of the transmission matrix, see Sec. IV.

In order to estimate the range of validity of the WA, we have also considered different superlattices. By decreasing the barrier width to $1 \mathrm{~nm}$, we have generated a strong coupling between the wells. Here the miniband width is slightly larger than the center of the miniband. Best agreement between the approaches is found for $w_{\text {eff }}=13.5 \mathrm{~nm}$. This value is larger than the one obtained above as the Wannier functions are less localized due to the small barrier width. As shown in Figs. 6(e) and (f) the results from the WA deviate clearly from the "exact" result in this case.

Finally, we considered the case of a larger well width, 15 $\mathrm{nm}$. The calculated miniband width $\left(4 T_{1}\right)$ is about $75 \%$ of the the center of the miniband $E^{a}$. Nevertheless, the agreement between both approaches is still satisfactory for low biases, as shown in Fig. 6(g). The second miniband extends from 44.8 to $84.7 \mathrm{meV}$ in this case. Its influence can be seen in the integrated transmission, Fig. 6(h). For $|U|>0.024 \mathrm{~V}$ the applied bias is larger than the gap between the lowest and second miniband. Then the coupling between the bands becomes important and the integrated transmission increases with bias for $|U|>0.03 \mathrm{~V}$ for the calculation in the discrete basis. Naturally this effect is not accounted for in the Wannier approximation due to the restriction to the lowest miniband.

In conclusion, we find that the Wannier approximation together with the wide-band limit from Secs. III A and III B gives good results if the miniband width is smaller than the energy of the center of the miniband and the applied bias is smaller than the gap between the minibands.

*Present and permanent address.

${ }^{1}$ L. Esaki and R. Tsu, IBM J. Res. Dev. 14, 61 (1970).

${ }^{2}$ A.Y. Shik, Sov. Phys. Semicond. 8, 1195 (1975) [Fiz. Tekh. Poluprovodn. 8, 1841 (1974)].

${ }^{3}$ Semiconductor Superlattices, edited by H. T. Grahn (World Scientific, Singapore, 1995).

${ }^{4}$ E. E. Mendez, in Proceedings of the 20th International Conference on the Physics of Semiconductors, edited by E. M. Anastassikas and J. D. Joannopoulos (World Scientific, Singapore, 1990), p. 1206.

${ }^{5}$ A. Sibille, J.F. Palmier, H. Wang, and F. Mollot, Phys. Rev. Lett. 64, 52 (1990).

${ }^{6}$ K. Leo, Phys. Scr. T68, 78 (1996).

${ }^{7}$ C.H. Kuan, D.C. Tsui, and K.K. Choi, Appl. Phys. Lett. 61, 456 (1992).

${ }^{8}$ C. Rauch, G. Strasser, K. Unterrainer, E. Gornik, and B. Brill, Appl. Phys. Lett. 70, 649 (1997).

${ }^{9}$ C. Rauch, G. Strasser, K. Unterrainer, W. Boxleitner, and E. Gornik, Phys. Status Solidi B 204, 393 (1997).

${ }^{10}$ C. Rauch, G. Strasser, K. Unterrainer, W. Boxleitner, E. Gornik, and A. Wacker, Phys. Rev. Lett. 81, 3495 (1998).

${ }^{11}$ A. Wacker, S. Bose, C. Rauch, G. Strasser, and E. Gornik, Superlattices Microstruct. 25, 43 (1999).

${ }^{12}$ F. Capasso, J. Faist, C. Sirtori, and A.Y. Cho, Solid State Commun. 102, 231 (1997).

${ }^{13}$ R. Tsu and L. Esaki, Appl. Phys. Lett. 22, 562 (1973).

${ }^{14}$ E.N. Glytsis, T.K. Gaylord, and K.F. Brennan, J. Appl. Phys. 70, 3920 (1991).

${ }^{15}$ E. Diez, A. Sánchez, and F. Domínguez-Adame, Phys. Rev. B 50, 14359 (1994).

${ }^{16}$ D. Z. Ting and T. C. McGill, in Quantum Transport in Ultrasmall Devices, edited by D. K. Ferry, H. L. Grubin, C. Jacoboni, and A. P. Jauho (Plenum Press, New York, 1995), p. 417.

${ }^{17}$ C. Caroli, R. Combescot, P. Nozieres, and D. Saint-James, J. Phys. C 4, 916 (1971).

${ }^{18} \mathrm{~S}$. Datta, Electronic Transport in Mesoscopic Systems (Cambridge University Press, Cambridge, 1995).

${ }^{19}$ R. Lake, G. Klimeck, R.C. Bowen, and D. Jovanovic, J. Appl. Phys. 81, 7845 (1997).

${ }^{20}$ Y. Meir and N.S. Wingreen, Phys. Rev. Lett. 68, 2512 (1992).

${ }^{21}$ A.-P. Jauho, N.S. Wingreen, and Y. Meir, Phys. Rev. B 50, 5528 (1994).

${ }^{22}$ H. Haug and A.-P. Jauho, Quantum Kinetics in Transport and Optics of Semiconductors (Springer, Berlin, 1996).

${ }^{23}$ L.V. Keldysh, Sov. Phys. JETP 20, 1018 (1965) [Zh. Eksp. Teor. Fiz. 47, 1515 (1964)].

${ }^{24}$ M. Büttiker, in Electronic Properties of Multilayers and LowDimensional Semiconductor Structures, edited by J. M. Chamberlain, L. Eaves, and J.-C. Portal (Plenum, New York, 1990), p. 51. 
${ }^{25}$ S. Doniach and E. H. Sondheimer, Green's Functions for Solid State Physicists (Benjamin, Reading, MA, 1974).

${ }^{26}$ W. Kohn, Phys. Rev. 115, 809 (1959).

${ }^{27}$ A. Wacker, in Theory of Transport Properties of Semiconductor Nanostructures, edited by E. Schöll (Chapman and Hall, London, 1998), Chap. 10.

${ }^{28}$ S.M. Goodnick, D.K. Ferry, C.W. Wilmsen, Z. Liliental, D. Fathy, and O.L. Krivanek, Phys. Rev. B 32, 8171 (1985).

${ }^{29}$ B. K. Ridley, in Theory of Transport Properties of Semiconductor Nanostructures (Ref. 27), Chap. 11.

${ }^{30} \mathrm{We}$ restrict ourselves to the lowest subband here. Matrix elements for intersubband scattering have been derived in: $\mathrm{Z}$. Tešanović, M.V. Jarić, and S. Maekawa, Phys. Rev. Lett. 57, 2760 (1986).
${ }^{31}$ F. Große and R. Zimmermann, Superlattices Microstruct. 17, 439 (1995).

${ }^{32}$ J. Grenzer, E. Schomburg, I. Lingott, A.A. Ignatov, K.F. Renk, U. Pietsch, U. Zeimer, B.J. Melzer, S. Ivanov, S. Schaposchnikov, P.S. Kopev, D.G. Pavelev, and Y. Koschurinov, Semicond. Sci. Technol. 13, 733 (1998).

${ }^{33}$ D. C. Langreth, in Linear and Nonlinear Electron Transport in Solids, edited by J. T. Devreese and V. E. van Doren (Plenum Press, New York, 1976).

${ }^{34}$ A. Wacker, A.-P. Jauho, S. Rott, A. Markus, P. Binder, and G. Döhler, Phys. Rev. Lett. 83, 836 (1999).

${ }^{35}$ J. Leo and A.H. MacDonald, Phys. Rev. B 43, 9763 (1991).

${ }^{36}$ P. Johansson, Phys. Rev. B 46, 12865 (1992).

${ }^{37}$ D.S. Fischer and P.A. Lee, Phys. Rev. B 23, 6851 (1981). 